\title{
Altered levels of plasma chemokines in breast cancer and their association with clinical and pathological characteristics
}

\author{
D. NARITA ${ }^{1,2}$, E. SECLAMAN ${ }^{1}$, A. ANGHEL ${ }^{1}$, R. ILINA ${ }^{3}$, N. CIREAP ${ }^{3}$, S. NEGRU ${ }^{4}$, I. O. SIRBU ${ }^{1}$, S. URSONIU ${ }^{5, *}$, C. MARIAN MR $^{1, *}$
}

${ }^{1}$ Department of Biochemistry, University of Medicine and Pharmacy "Victor Babes", Timisoara, Romania; ${ }^{2}$ Institute for Laboratory Diagnostic and Transfusion Medicine, Donauisar Klinikum, Deggendorf, Germany; ${ }^{3}$ Department of Surgical Oncology, University of Medicine and Pharmacy "Victor Babes", and Municipal Hospital, Timisoara, Romania; ${ }^{4}$ Department of Oncology, University of Medicine and Pharmacy "Victor Babes", and Oncomed Oncology Practice, Timisoara, Romania; ${ }^{5}$ Department of Public Health, University of Medicine and Pharmacy "Victor Babes", Timisoara, Romania

*Correspondence: sursoniu@umft.ro,cmarian@umft.ro

Received May 20, 2015 / Accepted August 17, 2015

Chemokines are a family of small, structurally related cytokines with chemoattractant and activation properties. In breast cancer, both epithelial cancer cells and cells within the microenvironment secrete chemokines with either tumor-promoting or anti-malignant potential. The equilibrium between these two chemokine activities plays a key role in the biology of the developing tumor, including its ability to metastasize. Here we evaluated the expression of chemokines in breast tumors and the plasma of breast cancer patients before treatment in order to identify a blood-based signature that could distinguish between malignant and non-malignant processes.

We screened the mRNA expression of chemokine genes using cDNA microarray on homogenous, laser-capture microdissected breast cancer specimens. Further, using a protein array approach, we determined the levels of selected chemokines in the plasma of patients with breast cancer, benign breast tumors and healthy women. Finally, we analyzed the association between the levels of chemokines in breast and blood samples with the pathological characteristics of the disease.

At mRNA level, 27 chemokines and 11 chemokine receptors were differentially expressed in cancers when compared with normal breast tissue. When compared to benign tumors, the only chemokine significantly upregulated in cancers was CXCL10. At protein level, with the exception of CXCL13, nine out of the ten selected chemokines (CCL2, CCL7, CCL18, CCL22, CXCL8, CXCL9, CXCL10, CXCL11 and osteoprotegerin) were significantly overexpressed in the plasma of breast cancers patients compared to healthy controls. After grouping, CXCL8, CXCL9 and CCL22 proved to be significant predictors for breast cancers as compared to healthy controls in a model of logistic regression. We found upregulation of CXCL8, CXCL11 and CXCL9 in triple negative carcinomas, CXCL9 in low proliferative carcinomas, and CXCL10, CCL7 and osteoprotegerin in poorly differentiated carcinomas. Furthermore, CXCL9 was overexpressed in lymph node negative tumors, whereas CXCL8 and CCL18 were higher in advanced stage carcinomas.

We identified a panel of chemokines dysregulated in breast cancer that could be further investigated as prospective novel diagnostic markers or for therapeutic and prognostic applications.

Key words: breast cancer, chemokines signature, protein array

\footnotetext{
Abbreviations: CCL - CC chemokine ligands; CCL18/PARC/MIP-4 pulmonary and activation regulated chemokine/macrophage inflammatory protein-4; CCL2/MCP-1 - monocyte chemotactic protein-1; CCL22/MDC - macrophage-derived chemokine; CCL7/MCP-3 - monocyte chemotactic protein-3; CCR - C-C chemokine receptor; CI: confidence interval; CXCL - Chemokine (C-X-C motif) ligand; CXCL10/IP-10 - interferon-gammainducible protein 10; CXCL11/I-TAC - interferon - inducible T-cell alpha chemoattractant; CXCL13/CXCR5/BLC - B lymphocyte chemoattractant;
}

CXCL9/MIG - monokine induced by interferon gamma; DNA dezoxiribonucleic acid; ER - estrogen receptor; HER2/ERBB2: human epidermal growth factor receptor 2; IFN $\gamma$ - interferon gamma; IL-8/ CXCL8 - interleukin 8; mRNA - messenger ribonucleic acid; OR: odd ratio; Osteoprotegerin/OPG/TNFRSF11B - osteoclastogenesis inhibitory factor/ tumor necrosis factor receptor superfamily member 11B; PR - progesterone receptor; TNFa - Tumor necrosis factor alpha. 
In Europe, breast cancer is the most frequent malignancy in women, with an incidence of 66.7/100000 (89.7/100000 in Western Europe) and a mortality of $17 \%$ (71.5\% in Western Europe), due in a vast majority to metastasis, the dissemination of the tumors at distance from the original location $[1$, 2]. Metastasis is regulated at least partially by the chemokinechemokine receptor interactions. To date, at least 48 human chemokines have been described, many of which bind to several of the 21 described human chemokine G-protein coupled receptors; conversely, some chemokine receptors bind multiple chemokines, indicating the existence of a complex, partially redundant signaling network [3].

Table 1. Characteristics of breast cancer patients included in the study

\begin{tabular}{|c|c|c|}
\hline \multirow{2}{*}{ Characteristics } & \multicolumn{2}{|c|}{ Breast Cancers $n=102(100 \%)$} \\
\hline & $\mathbf{n}$ & Percent \\
\hline \multicolumn{3}{|c|}{ Age (range between 35 - 90, Mean: 60.42, Median: 60) } \\
\hline$\leq 50$ & 17 & 16.67 \\
\hline$>50$ & 85 & 83.33 \\
\hline \multicolumn{3}{|l|}{ Tumor size $(\mathrm{cm})$} \\
\hline$<5$ & 68 & 66.67 \\
\hline$\geq 5$ & 34 & 33.33 \\
\hline \multicolumn{3}{|l|}{ Nodal status } \\
\hline Positive & 59 & 57.84 \\
\hline Negative & 43 & 42.16 \\
\hline \multicolumn{3}{|l|}{ Histology } \\
\hline Invasive Ductal & 73 & 71.57 \\
\hline Other types ${ }^{1}$ & 29 & 28.43 \\
\hline \multicolumn{3}{|l|}{ Histological grade $(\mathrm{G})$} \\
\hline G 1 & 6 & 5.88 \\
\hline G 2-3 & 96 & 94.12 \\
\hline \multicolumn{3}{|l|}{ Stage } \\
\hline Early (I, II, IIIA) & 68 & 66.67 \\
\hline Advanced (IIIB, IV) & 34 & 33.33 \\
\hline \multicolumn{3}{|l|}{ Estrogen receptor status } \\
\hline Positive & 79 & 77.45 \\
\hline Negative & 23 & 22.55 \\
\hline \multicolumn{3}{|c|}{ Progesterone receptor status } \\
\hline Positive & 61 & 59.80 \\
\hline Negative & 41 & 40.20 \\
\hline \multicolumn{3}{|l|}{ HER2/neu status } \\
\hline Negative $(0,+1)$ & 90 & 88.24 \\
\hline Positive $(+2,+3)$ & 12 & 11.76 \\
\hline \multicolumn{3}{|l|}{ Ki67 (\%) } \\
\hline$<20 \%$ & 39 & 38.24 \\
\hline$\geq 20 \%$ & 63 & 61.76 \\
\hline \multicolumn{3}{|l|}{ Obesity (BMI $\geq 30$ ) } \\
\hline Present & 68 & 66.67 \\
\hline Absent & 34 & 33.33 \\
\hline
\end{tabular}

${ }^{1}$ Mixed (ductal and lobular) (17), Lobular (3), Atypical Medullary (3), Medullary (1),

Invasive Papillary (1), Colloid (1), Metaplastic squamous (1), Anaplastic (1), DCIS (1)
Chemokines are a family of small, structurally related secreted cytokines with chemoattractant and activation properties that coordinate the homing of various subsets of haematopoietic cells to specific anatomical sites [4]. However, a growing body of evidence indicates that they also play an important role in tumor biology through modulation of biological processes such as migration, adhesion, apoptosis or proliferation, which further influence tumor growth, angiogenesis, local invasion and metastasis $[3,5]$. In breast cancer, both the epithelial cancer cells and the cells within the microenvironment secrete either tumor-promoting or anti-malignant chemokines, the equilibrium between these playing a key role in dictating the fate of the developing tumor and its ability to metastasize [6-8].

In the present study we evaluated the expression of chemokines in malignant cells and plasma of breast cancer patients before the onset of any treatment, with the aim to identify a blood-based chemokines signature that could discriminate between women with and without breast cancer. In this regard, we first screened by cDNA microarray the expression of chemokine genes in homogenous, laser-microdissected breast cancer epithelial cells as compared to normal mammary tissues. Using a protein array approach, we further examined a selected group of chemokines in the plasma of breast cancer patients, in comparison with patients with benign breast tumors and healthy volunteers. Finally, we investigated the chemokines levels association with several clinical and pathological characteristics known to be prognostic markers for breast cancer.

\section{Patients and methods}

Patients and tumor characteristics. We collected plasma samples (prior to surgery) and breast cancer tissue samples from 102 patients with malignant breast tumors and 42 patients with benign breast tumors admitted during 2009 and 2010 at the University Clinic of Surgical Oncology in Timisoara, Romania. Corresponding adjacent normal tissues from the same breast were also collected. The breast tissue samples were macroscopically and microscopically evaluated by a certified pathologist to confirm cancer, benign or normal status. Clinical and pathological characteristics of the patients were extracted from their medical records. We also collected plasma from 55 healthy age matched female volunteers, not pregnant and with no personal history of cancer and acute or chronic infections. Informed consent was obtained from all subjects, and the study was approved by the ethics committee of the institution. Table 1 summarizes the characteristics of breast cancer patients included in our study.

Gene expression analysis of chemokines. To identify the chemokine genes with altered expression in breast cancer, we performed cDNA microarray on homogenous, laser-capture microdissected breast cancer specimens. The specimens were chosen to represent the common breast cancer subtypes and they were classified as: 1) ER/PR positive, HER2/neu negative, 
low proliferative (Ki67=20\%) (luminal A), 2) ER/PR positive, HER2/neu negative, highly proliferative (Ki67=40\%) (luminal B), 3) ER/PR negative and HER2/neu positive, Ki67 $=10 \%$ (HER2/neu subtype), 4) triple negative, relapsed breast cancer with abundant inflammatory infiltrate and Ki67 $=15 \%$ (basallike), 5) triple negative infiltrative ductal carcinoma (IDC) of the breast, highly proliferative (Ki67=65\%) in premenopausal patient, (basal-like) and 6) triple negative, highly proliferative (Ki67 $=60 \%)$, medullary breast cancer, in postmenopausal patient. The breast cancer specimens were paired with normal, adjacent non-tumor tissues from the same patients, in order to evaluate the differential gene expression between pairs (tumor $v s$. normal tissues).

Samples preparation. Samples for gene expression were obtained after surgical resection and macroscopical assessment by a certified pathologist. Tissue samples were preserved in tubes with RNAlater (Ambion, Applied Biosystems) for 24 hours at $+4^{\circ} \mathrm{C}$ and then frozen at $-80^{\circ} \mathrm{C}$ until further use. Corresponding normal tissue removed from the same specimen was handled in similar manner.

Laser captured microdissection (LCM) and RNA extraction. Laser-capture microdissection was used to select and collect only the desired cell types (malignant groups of cells or normal mammary acini), under direct microscopic visualization, using an UV cutting system (MMI SmartCut Plus, MMI Molecular Machines and Industries, Glattburg, Switzerland) attached to an Olympus microscope. Following the manufacturer's protocol, frozen tissues were embedded in TissueTek medium and cut at $-30^{\circ} \mathrm{C}$ (Leica CM1850 cryostat, Leica Microsystems GmbH, Wetzlar, Germany). The $4 \mu \mathrm{m}$ cryosections were mounted on RNase free MMI MembranSlides, (MMI, Glattburg, Switzerland). The slides were immediately processed or stored at $-80^{\circ} \mathrm{C}$. Consecutive cryosections from each specimen were mounted also on silanized glass slides and, after standard hematoxylin and eosin staining (H\&E), the sections were evaluated by an experienced pathologist. The membrane slides for LCM were stained using an H\&E staining kit for LCM (MMI, Switzerland) following the manufacturer's protocol. LCM was performed immediately after staining. The selected cells were cut using adequate power and focus for UV laser shots and the cut areas were captured and placed in RNase free microcentrifuge tubes. RNA was extracted with RNAqueous-Micro kit (Ambion, Applied Biosystems, Germany) following manufacturer's specifications for microdissected cells. RNA concentration and purity were quantified on a NanoDrop ND1000 and the quality of RNA was evaluated on an Agilent 2100 Bioanalyzer (Agilent Technologies, Massy, France). RNA was stored at $-80^{\circ} \mathrm{C}$ until further gene expression analyses.

Using LCM, we obtained a quantity of RNA ranging between $2.1-17.7 \mathrm{ng} / \mu \mathrm{l}$ (average of 8.33 and median of 7.06 $\mathrm{ng} / \mu \mathrm{l})$, concentrated in $20 \mu \mathrm{l}$ elution solution, with A260/280 between 1.86 and 2.11 (average of 1.95 and median of 1.96). The RIN (RNA integrity number) generated by the Agilent 2100 Bioanalyzer was between 7.4 and 8.5 for the majority of LCM samples. The LCM samples with RIN below 7 (four samples) were excluded.

DNA microarray analysis. cDNA microarray analysis was performed using the Human GE 4x44K v2 Microarray Kit, $4 \mathrm{x} 44 \mathrm{~K}$ (Agilent Technologies, Inc, Santa Clara, USA) following the manufacturer's protocol. This array targets 19596 Entrez Gene RNAs of which 197 are chemokine related genes. Briefly, Agilent's Low Input Quick Amp Labeling Kit was used with 10ng sample input RNA for one-color processing to synthesize cDNA with the help of AffinityScript-reverse transcriptase and oligo dT-promoter primers. The antisense cRNA was then purified (Agilent Absolute RNA Nanoprep kit) and quantified in order to determine the yield and specific activity of each reaction, namely $\mu \mathrm{gcRNA}$ yield and specific activity (pmol Cy3 per $\mu \mathrm{g}$ cRNA). The microarray slides were then blocked and prepared for hybridization, hybridized for $17 \mathrm{~h}$, washed, stabilized and scanned on the green dye channel, with $5 \mu \mathrm{m}$ resolution (Agilent microarray scanner G2565BA), using the manufacturer's instructions and reagents. Feature Extraction software 9.5 was used for data extraction. The data were analyzed with the aid of GeneSpring GX v.10.0 software.

\section{Chemokine analysis at protein level}

Sample preparation. For plasma chemokine concentrations assessment, peripheral venous blood $(3 \mathrm{ml})$ was collected using EDTA as anticoagulant between 8 and 9 a.m. Within 30 minutes after collection, samples were centrifuged for $5 \mathrm{~min}$ at $3500 \mathrm{x}$; plasma was immediately separated, aliquoted and stored in a $-80^{\circ} \mathrm{C}$ freezer until further analyses.

Protein array analysis. Levels of plasma chemokines were determined using a quantitative protein array platform (Quantibody array) with 10-multiplexed, customized chemokines according to the manufacturer's (Raybiotech, Inc, USA) protocol. The Quantibody ${ }^{\circ}$ array multiplexed sandwich ELISA-based technology uses a pair of specific antibodies, as follows: the capture antibody is first bound to the glass surface and after overnight incubation with samples or standards $(100 \mu \mathrm{l}$ each), the target chemokine is trapped on the solid surface. The second, biotin-labeled detection antibody (which can recognize a different epitope of the target chemokine) is then added and the chemokine-antibody-biotin complex was visualized after the addition of the streptavidin-labeled Cy3 equivalent dye using a laser scanner (SpotLight Scanner, Arrayit Corporation, Sunnyvale, US). The expected sensitivity is as low as $1 \mathrm{pg} / \mathrm{ml}$ with a $\mathrm{CV}<20 \%$ and less than $1 \%$ cross-reactivity.

Data were extracted using GenePix microarray analysis software (Molecular Devices, Inc., Sunnyvale, California, US) and analyzed quantitatively with the Quantibody ${ }^{\circledR} \mathrm{Q}$-Analyzer software, an array specific program provided by the manufacturer (Raybiotech, Inc., US). The chemokines concentration in the samples was interpolated from standard curves generated using serial dilutions of the respective recombinant proteins provided by the manufacturer.

Statistical analysis. Descriptive statistics, including median, mean and standard deviation were computed for each of the 
Table 2. Differential levels of chemokine genes expression (as compared with the non-tumor adjacent tissues)

\begin{tabular}{|c|c|c|c|c|c|c|}
\hline \multirow[t]{2}{*}{ Gene } & \multicolumn{6}{|c|}{$\begin{array}{l}\text { Breast cancer subtypes, median value of differential } \\
\text { expression }\end{array}$} \\
\hline & S1 & S2 & S3 & S4 & S5 & S6 \\
\hline CXCL10 & 95.92 & 0.35 & -0.39 & 5.56 & 2.78 & 1.36 \\
\hline CXCL11 & 86.94 & 0.68 & -0.08 & 5.47 & 2.96 & 1.19 \\
\hline TNFRSF11B & 63.60 & 0.16 & 3.12 & -0.07 & 1.14 & 5.13 \\
\hline CCL7 & 26.81 & 0.09 & 1.47 & 2.20 & -0.01 & 20.23 \\
\hline IL4I1 & 19.24 & 0.73 & 1.55 & 3.73 & 3.32 & 2.79 \\
\hline CXCL1 & 14.46 & -0.32 & -0.75 & -0.74 & -0.85 & -0.63 \\
\hline IL23A & 11.80 & 0.59 & -0.03 & 0.42 & 0.46 & -0.33 \\
\hline CCL3 & 11.75 & 0.06 & 6.62 & 4.96 & 1.06 & 1.70 \\
\hline IL8 & 11.74 & 0.14 & 1.05 & 0.73 & -0.09 & 1.88 \\
\hline CXCR4 & 11.64 & 0.45 & 2.81 & 2.26 & 2.45 & 0.68 \\
\hline CXCL9 & 11.25 & 0.39 & -0.31 & 1.83 & 4.45 & 0.16 \\
\hline CCR7 & 11.20 & 0.45 & 1.01 & 0.97 & 4.74 & -0.28 \\
\hline IL1b & 11.03 & 0.16 & 0.29 & 1.06 & -0.25 & -0.02 \\
\hline $\mathrm{TNFa}$ & 9.36 & 0.55 & -0.60 & 0.60 & 1.00 & -0.40 \\
\hline CCL4 & 8.97 & 0.42 & 2.25 & 2.40 & 2.24 & 0.20 \\
\hline CCL2 & 8.56 & 1.03 & 4.63 & 2.98 & 2.03 & 4.08 \\
\hline IL21R & 7.88 & 0.99 & 3.00 & 1.97 & 6.80 & 1.67 \\
\hline CCL3L3 & 7.87 & 0.12 & 4.15 & 3.65 & 0.88 & 1.41 \\
\hline IL8RBP & 7.55 & 0.39 & -0.40 & 0.03 & 0.60 & -0.41 \\
\hline CCL17 & 7.15 & -0.29 & -0.30 & 0.57 & 15.52 & -0.11 \\
\hline IL2RG & 6.85 & 0.51 & 0.20 & 0.27 & 3.32 & -0.21 \\
\hline IL2RB & 5.65 & 0.52 & 0.19 & 0.54 & 2.54 & -0.17 \\
\hline IL32 & 5.14 & 1.03 & 1.07 & 3.38 & 2.69 & 1.35 \\
\hline CCL18 & 5.06 & 1.30 & 7.26 & 0.65 & 15.25 & 8.99 \\
\hline CCR1 & 4.93 & 0.18 & 2.31 & 0.61 & 1.27 & 1.26 \\
\hline OSCAR & 4.22 & 0.09 & 2.11 & 0.62 & 0.32 & 2.26 \\
\hline CXCR5 & 3.85 & 0.16 & 1.71 & 0.07 & 7.42 & -0.29 \\
\hline CXCL13 & 3.70 & 0.32 & 0.40 & -0.55 & 6.38 & 19.21 \\
\hline CCL13 & 3.13 & -0.10 & 2.98 & 0.02 & 8.57 & 1.07 \\
\hline CCL19 & 3.06 & 0.14 & -0.31 & 0.03 & 5.32 & -0.76 \\
\hline IL18 & 2.99 & 0.73 & 1.47 & 0.73 & 2.44 & 1.91 \\
\hline IFNg & 2.88 & 0.20 & -0.09 & 0.39 & 1.21 & 0.00 \\
\hline IL2RA & 2.49 & 0.10 & 0.50 & 0.53 & 0.95 & 0.09 \\
\hline CCL26 & 1.38 & 0.03 & -0.23 & 0.38 & 4.89 & 0.18 \\
\hline CXCL17 & 0.52 & 0.28 & 0.17 & 0.59 & 29.99 & 8.42 \\
\hline CCL11 & 0.34 & 0.38 & 0.62 & 2.37 & 4.72 & 0.66 \\
\hline CCL22 & 0.10 & 0.29 & -0.09 & 0.11 & 0.16 & -0.14 \\
\hline IL17B & -0.94 & 6.80 & -0.81 & -0.90 & -0.90 & -0.89 \\
\hline
\end{tabular}

To identify a panel of cytokines that are overexpressed in breast cancer, we performed cDNA microarray on selected breast cancer specimens. The specimens were selected to represent the common breast cancer subtypes and they were classified as: $\mathrm{S} 1=$ Basal-like (triple negative, $\mathrm{CDI}$, premenopausal patient, highly proliferative): $\mathrm{S} 2=$ Luminal A (ER/PR positive, HER2/ neu negative, low proliferative); $S 3=$ Basal-like (triple negative, relapsed, Ki67=15\%); S4=Luminal B (ER/PR positive, HER2/neu negative, highly proliferative Ki67=40\%); S5= HER2/neu subtype (ER/PR negative and HER2/ neu positive, Ki67=10\%); S6= Basal-like (triple negative, Ki67=60\%, medullary breast cancer); the breast cancer specimens were paired with normal, adjacent non-tumor tissues from the same patients in order to evaluate the differential gene expression between pairs (tumor $v s$. normal tissues).

${ }^{*}$ Differentially expressed genes ( $>2$-fold) in the median value and FDR (false discovery rate) $<0.05$ in the investigated breast cancer subtypes as compared with the adjacent, normal breast tissues; the selected panel of 10 chemokines is displayed in bold. cytokines using Stata version 9.2 (Statacorp, Texas, USA). A two-sample, rank sum Wilcoxon (Mann - Whitney) test was used to determine differences in the median values and to compare groups. Logistic regression was used to determine how well the groups of chemokines were able to distinguish between women with or without cancer. Receiver operator characteristic (ROC) curve for the stepwise model of the best combination of chemokines was plotted and area under curve (AUC) was calculated. The ROC curve is a plot of the true positive rate (sensitivity) against the false positive rate (1 specificity) for the different possible cutpoints. The threshold for significance was set at $\mathrm{p}<0.05$.

\section{Results}

Gene expression analysis and chemokines selection. 27 chemokines (CCL2, CCL3, CCL4, CCL7, CCL11, CCL13, CCL17, CCL18, CCL19, CCL22, CCL26, CCL3L3, IL4L1, IL17 $\beta$, IL18, IL23 $\alpha$, IL32, IL1 $\beta$, Tumor necrosis factor alpha/ TNF $\alpha$, interferon gamma/IFN $\gamma$, CXCL1, CXCL8, CXCL9, CXCL10, CXCL11, CXCL13, CXCL17) and 11 chemokine receptors (tumor necrosis factor receptor superfamily member 11B/TNFESF11B or osteoprotegerin/OPG, osteoclast-associated immunoglobulin-like receptor/OSCAR, IL21R, IL2Ra, IL2R $\gamma$, IL2R $\beta$, IL8RBP, CXCR4, CXCR5, CCR1, CCR7) genes were found to be differentially expressed at $>2$-fold in the median value and FDR (false discovery rate) $<0.05$ in breast cancer epithelial cells as compared with the adjacent normal breast tissues, in at least one of the investigated samples (Table 2).

From these proteins, by searching the literature and commercially available kits, we selected 10 chemokines to be further tested at the protein level: IL-8 (CXCL8), CXCL9 (MIG, monokine induced by interferon gamma), CXCL10 (IP-10, interferon-gamma-inducible protein 10), CXCL11 (I-TAC, interferon - inducible T-cell alpha chemoattractant), CXCL13 (CXCR5 or BLC, B lymphocyte chemoattractant), CCL2 (MCP-1, monocyte chemotactic protein-1), CCL7 (MCP-3, monocyte chemotactic protein-3), CCL22 (MDC, macrophage-derived chemokine), osteoprotegerin (OPG or TNFRSF11B, osteoclastogenesis inhibitory factor, or tumor necrosis factor receptor superfamily member 11B) and CCL18 (PARC - pulmonary and activation regulated chemokine or MIP-4, macrophage inflammatory protein-4).

\section{Protein array analysis}

Chemokines plasma concentration in cancer patients vs healthy controls and benign tumor patients. With the exception of CXCL13, all of the investigated chemokines were upregulated in breast cancer patients in comparison with healthy controls ( $p$ ranges between 0.009 and $<0.001$ ) (Table 3). Plasma from breast carcinoma patients exhibited extremely high levels of CCL18, CXCL9, OPG and CXCL10. The greatest differences in expression between normal controls and carcinoma 
patients occurred for CCL18, CXCL11, CXCL10, CXCL8 and CCL2 ( $<<0.001)$.

With the exception of CCL7, CXCL13 and OPG, the plasma of benign breast tumor patients exhibited higher levels of chemokines than normal plasma, (p between 0.02 and $<0.001$ ). The CCL2 (range 126.9 - 705.8, median $587.5 \mathrm{pg} / \mathrm{ml}$ ) and CCL22 (49.1 - 2762.2, median $502.3 \mathrm{pg} / \mathrm{ml})$ proteins were also more abundant in the plasma of benign tumor patients as compared to breast cancer patients, but when compared to control samples, the differences did not reach statistical significance.

When we compared cancers with benign tumors, we observed that from the investigated panel, the only chemokine significantly upregulated was CXCL10 ( $\mathrm{p}=0.001)$.

The significant $p$ values for each cytokine investigate were included in a model of logistic regression and a stepwise approach was used to determine the combinations that might distinguish between women with and without breast cancer. A group of three chemokines, i.e. IL8/CXCL8, MIG/CXCL9 and MDC/CCL22, were found to significantly predict breast cancers when compared to healthy controls, with an area under the curve (AUC) of 0.777 . The odds ratios and their respective confidence intervals (OR; 95\%CI) for the three significant chemokines are as follows: IL8/CXCL8 (3.05; 1.39-6.71), MIG/ CXCL9 $(4.33 ; 1.91-9.80)$ and MDC/CCL22 (2.74; 1.27-5.87). The AUC for the associated Receiver Operated Characteristics (ROC) curve is plotted in Figure 1.

Association of plasma chemokines with clinicopathological parameters. The proportion of ER-negative, PR-negative, and HER $2 /$ neu positive $(2+$ and $3+$ ) tumors was $23 \%, 40 \%$ and $12 \%$ of the breast cancer patients, respectively (Table 1 ). We evaluated whether there was any association between chemokine levels and ER, PR and HER2/neu status. We observed that CXCL8 ( $\mathrm{p}=0.012)$, CXCL9 $(\mathrm{p}=0.007)$, CXCL11 $(\mathrm{p}=0.002)$ and CXCL13 ( $\mathrm{p}=0.041)$ were significantly overexpressed in ER-negative tumors compared with ER-positive ones. CXCL8 was also significantly overexpressed in PR-negative carcinomas $(p=0.019)$. As for the remaining chemokines,

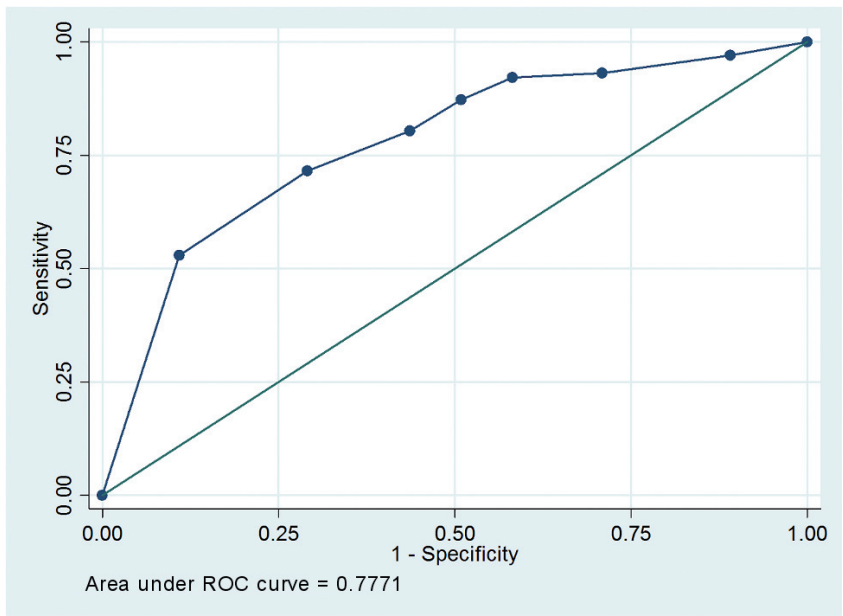

Figure 1. Receiver operator characteristic curve for the stepwise model of the best combination of chemokines (IL8/CXCL8, MIG/CXCL9 and MDC/CCL22).

although more abundantly expressed in ER-negative and PR-negative carcinomas, the differences were not statistically significant (Supplementary Table 1). Furthermore, CXCL9/ MIG was significantly inversely correlated with HER2 expression, being overexpressed $(p=0.016)$ in HER2-negative tumors. When we analyzed the distribution of chemokines by combining the three parameters (ER, PR and HER2), we found that CXCL8 ( $\mathrm{p}=0.017)$, CXCL11 $(\mathrm{p}=0.011)$ and CXCL9 $(\mathrm{p}=0.04 \mathrm{l})$ were significantly upregulated in triple negative breast carcinomas.

We further evaluated whether chemokine expression is associated to other clinicopathological parameters such as: tumor size, histopathological type, lymph node metastasis status, stage, histological grade (G), proliferation (Ki67), patient age or BMI (body mass index). The chemokines profile was not associated with patient age or BMI (data not shown). The proliferation rate was significantly associated $(\mathrm{p}=0.012)$

Table 3. Plasma chemokine levels in cancer and benign tumor patients and normal controls

\begin{tabular}{|c|c|c|c|c|c|c|}
\hline Chemokine & $\begin{array}{c}\text { Normal } \\
(\mathrm{n}=55)\end{array}$ & $\begin{array}{l}\text { Benign } \\
(\mathrm{n}=41)\end{array}$ & $\begin{array}{c}\text { Cancer } \\
(n=102)\end{array}$ & $\begin{array}{c}\text { p Cancer } \\
\text { vs. } \text { Normal }\end{array}$ & $\begin{array}{c}\text { p Benign } \\
\text { vs. Normal }\end{array}$ & $\begin{array}{c}\text { p Cancer } \\
\text { vs. Benign }\end{array}$ \\
\hline MCP-1/CCL2 & $237.90(2.2-1432.5)$ & $587.50(126.9-705.8)$ & $502.30(49.1-2762.2)$ & $<0.001$ & 0.002 & 0.623 \\
\hline MCP-3/CCL7 & $4.00(0.7-589.1)$ & $7.10(0.1-1200.3)$ & $26.80(0.1-573.3)$ & 0.002 & 0.099 & 0.102 \\
\hline MDC/CCL22 & $1468.30(0.1-12573.8)$ & $6584.70(1523.7-12985.9)$ & $5649.00(88.5-65934.6)$ & $<0.001$ & $<0.001$ & 0.466 \\
\hline IL8/CXCL8 & $3.10(0.1-160.2)$ & $7.90(0.5-299.1)$ & $9.05(0.1-1452.2)$ & $<0.001$ & $<0.001$ & 0.457 \\
\hline MIG/CXCL9 & $9286.90(2147.5-43127.1)$ & $12587.90(5284.5-55328.6)$ & $16411.40(470.0-244790.2)$ & $<0.001$ & 0.006 & 0.517 \\
\hline IP-10/CXCL10 & $354.70(0.1-2445.4)$ & $538.40(126.9-2593.5)$ & $945.15(18.5-15275.0)$ & $<0.001$ & 0.005 & 0.001 \\
\hline I-TAC/CXCL11 & $49.80(13.4-700.4)$ & $145.60(10.5-2112.6)$ & $227.50(0.1-8960.1)$ & $<0.001$ & 0.005 & 0.308 \\
\hline BLC/CXCL13 & $312.60(2.5-4786.4)$ & $434.50(99.4-4156.4)$ & $525.00(12.7-10530.1)$ & 0.103 & 0.317 & 0.264 \\
\hline OPG/TNFRSF11B & $659.50(71.4-6921.4)$ & $986.50(47.6-3769.6)$ & $1441.30(48.1-27646.4)$ & 0.009 & 0.130 & 0.408 \\
\hline PARC/CCL18 & $28735.3(9641.03-58748.6)$ & $34621.30(15487.6-89654.3)$ & $42928.95(3047.0-242698.0)$ & $<0.001$ & 0.020 & 0.169 \\
\hline
\end{tabular}

Values are expressed as median (range; $\mathrm{pg} / \mathrm{ml}$ ) 
with CXCL9, which was more abundant in low proliferative carcinomas as determined using Ki67. MCP-1, MCP-3, CXCL8, CXCL10 and OPG were more abundant in highly proliferative tumors, but the differences were not statistically significant. With respect to the histological grade, CXCL10/IP$10(\mathrm{p}=0.010), \mathrm{CCL} 7 / \mathrm{MCP}-3(\mathrm{p}=0.043)$ and OPG/TNFRSF11B ( $\mathrm{p}=0.048$ ), were significantly overexpressed in poorly differentiated carcinomas; MCP-1, MDC, CXCL8, CXCL13 and OPG were also overexpressed in high grade carcinomas (G2, G3) but the difference was not statistically significant.

CXCL9/MIG was significantly overexpressed in lymph node negative tumors ( $\mathrm{p}=0.035)$ while CCL22/MDC, CXCL8/ IL8, CXCL10, CXCL13/BLC, OPG and CCL18/PARC, although more abundant in lymph node positive tumors, did not reach statistical significance.

When the chemokine profile was analyzed according to tumor stage, although expressed at higher levels in advanced stage cancers, CCL2, CCL22, CXCL10, CXCL13 and OPG did not reach statistically significance. MCP- $1(\mathrm{p}=0.040)$ and CXCL10 $(\mathrm{p}=0.010)$ were overexpressed in larger $(\geq 5 \mathrm{~cm})$ tumors. Regarding the histological type, we observed a statistically significant association ( $\mathrm{p}=0.027$ ) only for CXCL10 which was more abundant in invasive ductal carcinomas.

\section{Discussion}

To gain insights regarding chemokines implication in breast cancer, we analyzed malignant and corresponding normal adjacent tissue samples from patients with different subtypes of breast cancer, assessing their chemokines gene expression profile on homogenous, laser-capture microdissected breast cancer epithelial cells. Using this approach, we identified a panel of 27 chemokines and 11 chemokine receptors that were differentially expressed in breast cancers as compared with the adjacent normal breast tissues (Table 2). From this panel, we selected ten chemokines to be quantified using a quantitative protein array platform, in the plasma of breast cancer patients, benign breast tumor patients and healthy controls.

Nine out of the ten investigated chemokines (CCL2, CCL7, CCL18, CCL22, CXCL8, CXCL9, CXCL10, CXCL11, OPG) were strongly overexpressed in the plasma of breast cancer patients as compared with healthy controls ( $p$ between 0.002 and $<0.001$ ). However, only CXCL10/IP-10 was significantly upregulated in cancers when compared with either benign tumors $(\mathrm{p}=0.001)$ or normal controls $(\mathrm{p}<0.001)$. We further evaluated whether the chemokine expression profile could be correlated with the clinicopathological parameters linked to prognostic such as ER, PR, HER2 expression, tumor size, histopathological type, lymph node metastasis status, stage, histological grade $(\mathrm{G})$, proliferation (Ki67), patient age or BMI. We will emphasize below the selected chemokines that were investigated in our study and we will highlight and discuss the associations that were observed.

CXCL8/IL-8 is a prototypical member of a superfamily of small, inducible, ELR-positive CXCs chemokines, originally identified as monocyte derived factors capable of attracting and activating neutrophils [9]. CXCL8 acts as an autocrine growth factor for tumors [6], and like other ELR-positive CXC chemokines it promotes angiogenesis [10]. IL-8 has also been shown to stimulate self-renewal of breast cancer stem cell in vitro and to render tumors resistant to chemotherapy [11]. In our study, IL-8 was upregulated in ER- and PR-negative tumors $(p=0.012$ and $p=0.019)$. Although not statistically significant, this chemokine was also more abundant in the following types of carcinomas: poorly differentiated, highly proliferative, nodal metastasis, high size, HER2-positive. Consistent with our data, a number of studies suggest that IL-8 is involved in breast cancer invasiveness, angiogenesis and metastasis [12, 13]. In other studies, CXCL8 was also correlated with the histological grade [14, 15], and macrophage infiltration [14], while a high CXCL8 level in metastatic breast cancers was linked to osteoclastogenesis and bone resorbtion, leading to an enhanced osteolysis [16].

CXCL10 also known as small-inducible cytokine B10 was shown to possess both tumor-inhibitory [17], and tumor growth stimulatory properties [18]. It has been also shown that CXCL10 may promote tumor cell proliferation and invasion $[19,20]$. This controversy in the function of CXCL10 could be related to the observation that the CXCL10 receptor (CXCR3) is alternatively spliced in different human tissues to produce two known variants, CXCR3-A and CXCR3-B [21, 22]. CXCL10 binding to CXCR3-A leads to cell proliferation and chemotaxis, whereas binding of CXCL10 to CXCR3-B inhibits cell growth [23]. CXCR3 is also activated by three interferon (IFN)- $\gamma$-inducible ligands which can collaborate, but they also have non-redundant functions in vivo and even compete with each other [24]. Although CXCL9, CXCL10, and CXCL11 are known as members of the tumor-inhibitory IFN- $\gamma$-inducible CXC chemokines [25], they could also have divergent (pro- or anti-malignant) effects, depending on the CXCR3 variants expressed by the cancer cells [26]. In our samples, CXCL10/IP-10 was significantly upregulated in higher size $(\mathrm{p}=0.010)$, poorly differentiated $(\mathrm{p}=0.010)$ and ductal $(\mathrm{p}=0.027)$ carcinomas; although elevated, CXCL10 was not significantly associated with ER- and PR-negative, HER2positive, higher Ki67, lymph node positive and advanced stage carcinomas. Overall and in accordance with other studies [19, $23,27]$, our results suggest that this chemokine associates with an aggressive phenotype of breast carcinomas.

CXCL11/I-TAC was upregulated in ER-negative tumors $(\mathrm{p}=0.002)$ and, without reaching statistical significance, also in well differentiated, low Ki67, absent lymph node metastasis, lower size, early stage, HER2-positive ductal carcinomas. These results are in accordance with other studies that suggest that this chemokine, when overexpressed, could exert an antitumor effect and suggest a good prognosis [3].

In our study, CXCL9/MIG was more abundant in ERnegative tumors $(\mathrm{p}=0.007)$, HER2-negative $(\mathrm{p}=0.016)$, low proliferative carcinomas $(\mathrm{Ki} 67 \leq 20)(\mathrm{p}=0.012)$ and lymph node negative cancers $(\mathrm{p}=0.035)$ but also in well differentiated, 
small size, early stage and other than ductal type carcinomas, although without reaching a statistically significant level. These results are in accordance with other studies $[3,28]$ and suggest that this chemokine, when overexpressed, could select a subgroup of good prognostic ER-negative breast carcinomas. Overexpression of CXCL9 leads to a marked inhibition of both local and metastatic tumor growth, a therapeutic effect mediated by host T-cell and NK cell infiltration into the tumor [28].

Although in our study it was not significantly related to the clinicopathological parameters of breast cancers, OPG was more abundant in ER/PR-negative, HER2-positive, and highly proliferative, poorly differentiated, lymph node positive, higher size and advanced stage ductal carcinomas. Our results are in accordance with other studies that found OPG expression to be negatively correlated with increasing tumor grade and showed that OPG produced by breast cancer cells enhanced tumor cell survival by inhibiting TRAIL-induced apoptosis [29]. On the other hand, OPG treatment in animal models of breast cancer inhibited the development of osseous lesions and the growth of cancer cells in the bone [30]. Experimental data indicate that OPG could perhaps be a potential therapeutic option for the treatment of bone lyses observed in patients with metastatic breast cancer [31].

CCL22/MDC, a C-C motif chemokine secreted by dendritic cells and macrophages elicits its effects by interacting with cell surface chemokine receptors such as CCR4 [32]. In our study, CCL22 was not significantly related to ER/PR status or any other clinicopathological parameter, although it was more abundant in HER2-positive carcinomas, poorly differentiated, lymph node metastasized, advanced stage, and lower size carcinomas, suggesting a role for this chemokine in the early stage of tumor formation. Nevertheless, CCL22 was dramatically increased in the plasma of patients with benign tumors compared to normal controls. In vitro interaction between myeloid (monocytes and dendritic cells) and tumor cells leads to increased CCL22 production, suggesting that the initial immune cell infiltration might trigger the production of CCL22. This might explain not only the recruitment of regulatory $\mathrm{T}$ cells, but also their conventional silencing and prevention of activation further leading to, immune escape and ultimately to tumor progression [33].

CXCL13 (or B lymphocyte chemoattractant - BLC) is known to be expressed by stromal cells within B-cell follicles in secondary lymphoid tissues [34]. CXCL13 is selectively chemotactic for B cells and elicits its effects by interacting with chemokine receptor CXCR5 [35]. In our study, CXCL13/BLC was not statistically significant overexpressed in the plasma of breast cancers compared to benign tumors or normal patients but, in accordance with other studies [36, 37], it was significantly associated with ER-negative breast cancers and advanced stage breast carcinomas. CXCL13 was also overexpressed in high grade carcinomas (G3) and axillary lymph node metastasis, suggestive for an aggressive tumor biology. Panse et al. (2008) found that CXCL13 is overexpressed in breast cancer tissues at both mRNA and protein levels and that increased serum levels of this cytokine can be found in metastatic breast cancer patients; this indicates a possible role of CXCL13 in the development and progression of breast cancer [36]. Recently, CXCL13 was identified included in a set of 14 prognostic gene candidates as metastasis predictors in early stage hormone receptor-negative and triple-negative breast cancer [37].

CCL18 is predominantly produced by M2 phenotype monocyte-derived cells and its excessive production in M2 macrophages was demonstrated in various chronic inflammations [38]. However, the role of CCL18 in cancer progression is controversial. CCC18 was reported to participate in immunosuppression of ovarian cancer [39], but was also associated with prolonged survival in patients with gastric cancer [40]. Cytokine profile analysis of breast tumor-associated macrophages showed that CCL18 is abundantly expressed and promotes migration and invasion of breast cancer cells by enhancing their adherence to extracellular matrix; the CCL18 level in blood or cancer stroma was found to be associated with metastasis of patients with breast cancer [41].

CCL7/MCP-3 is a chemotactic factor that attracts monocytes and eosinophils (but not neutrophils), augments monocyte anti-tumor activity and binds to CCR1, CCR2 and CCR3 [42]. In our work, CCL7 was significantly upregulated in breast cancers compared with healthy women and it was correlated with high-grade (G3) cancers, but not with tumor size or proliferation rate, suggesting that, like CCL18, this chemokine could be related especially to tumor aggressiveness and not to tumor growth kinetics.

CCL2/MCP-1 is a potential monocyte-recruiting factor responsible for the high presence of tumor-associated macrophages in tumors [43]. Elevated levels of CCL2 were significantly correlated with early relapse, advanced tumor stage, the grade of breast tumors, lymph node metastasis [44] and with poor prognosis [14]. In our study, CCL2 was significantly correlated only with the size of the tumor. In accordance with some studies that suggest that under specific conditions CCL2 may have anti-tumor effects [45], we found an increased level of CCL2 in the plasma of patients with benign breast conditions.

In summary, the overexpression of CXCL8 and CXCL10 is clearly linked to certain negative prognostic markers (ER-negative, poorly differentiated, advanced stage ductal carcinomas). In addition, CXCL9 which is overexpressed in lymph node negative, low proliferative, well differentiated, small size, early stage and other than ductal type carcinomas, but also ER-negative and HER2-negative carcinomas could select a subgroup of good prognostic ER-negative breast carcinomas. Our model of logistic regression identified a group of three chemokines (CXCL8, CXCL9 and CCL22) with the potential to differentiate women with and without cancer. CXCL10 was the only chemokine found significantly upregulated in cancers when compared either with benign tumors or normal controls. In conclusion, we identified a relevant 
panel of chemokines that are clearly dysregulated in breast cancer and that could be further investigated for prospective novel prognostic markers or therapeutic approaches for breast cancer.

Supplementary information is available in the online version of the paper.

Acknowledgements: This study was supported by CNCSIS UEFISCSU, project number 1197/2009, PN II - IDEI, PCE, code $1750 / 2008$.

\section{References}

[1] AUTIER P., BONIOL M., LA VECCHIA C., VATTEN L., GAVIN A., et al. Disparities in breast cancer mortality trends between 30 European countries: retrospective trend analysis of WHO mortality database. BMJ 2010; 341(c3620.

[2] FERLAY J., SOERJOMATARAM I., DIKSHIT R., ESER S., MATHERS C., et al. Cancer incidence and mortality worldwide: sources, methods and major patterns in GLOBOCAN 2012. Int J Cancer 2015; 136: E359-386. http://dx.doi. org/10.1002/ijc.29210

[3] BEN-BARUCH A. The multifaceted roles of chemokines in malignancy. Cancer Metastasis Rev 2006; 25: 357-371. http:// dx.doi.org/10.1007/s10555-006-9003-5

[4] MULLER W. A. New mechanisms and pathways for monocyte recruitment. J Exp Med 2001; 194: F47-51. http://dx.doi. org/10.1084/jem.194.9.f47

[5] RAMAN D., BAUGHER P. J., THU Y. M. and RICHMOND A. Role of chemokines in tumor growth. Cancer Lett 2007; 256: 137-165. http://dx.doi.org/10.1016/j.canlet.2007.05.013

[6] KAKINUMA T. and HWANG S. T. Chemokines, chemokine receptors, and cancer metastasis. J Leukoc Biol 2006; 79: 639-651. http://dx.doi.org/10.1189/jlb.1105633

[7] ALI S. and LAZENNEC G. Chemokines: novel targets for breast cancer metastasis. Cancer Metastasis Rev 2007; 26: 401-420. http://dx.doi.org/10.1007/s10555-007-9073-Z

[8] LAZENNEC G. and RICHMOND A. Chemokines and chemokine receptors: new insights into cancer-related inflammation. Trends Mol Med 2010; 16: 133-144. http://dx.doi. org/10.1016/j.molmed.2010.01.003

[9] REED M. J. and PUROHIT A. Breast cancer and the role of cytokines in regulating estrogen synthesis: an emerging hypothesis. Endocr Rev 1997; 18: 701-715. http://dx.doi. org/10.1210/edrv.18.5.0314

[10] STRIETER R. M., POLVERINI P. J., KUNKEL S. L., ARENBERG D. A., BURDICK M. D., et al. The functional role of the ELR motif in CXC chemokine-mediated angiogenesis. J Biol Chem 1995; 270: 27348-27357. http://dx.doi.org/10.1074/jbc.270.45.27348

[11] VISVADER J. E. and LINDEMAN G. J. Cancer stem cells in solid tumours: accumulating evidence and unresolved questions. Nat Rev Cancer 2008; 8(10): 755-768. http://dx.doi. org/10.1038/nrc2499

[12] VAZQUEZ-MARTIN A., COLOMER R. and MENENDEZ J. A. Protein array technology to detect HER2 (erbB-2)-induced ,cytokine signature` in breast cancer. Eur J Cancer 2007; 43: 1117-1124. http://dx.doi.org/10.1016/j.ejca.2007.01.037

[13] LIN Y., HUANG R., CHEN L., LI S., SHI Q., et al. Identification of interleukin-8 as estrogen receptor-regulated factor involved in breast cancer invasion and angiogenesis by protein arrays. Int J Cancer 2004; 109: 507-515. http://dx.doi.org/10.1002/ ijc. 11724

[14] CHAVEY C., BIBEAU F., GOURGOU-BOURGADE S., BURLINCHON S., BOISSIERE F., et al. Oestrogen receptor negative breast cancers exhibit high cytokine content. Breast Cancer Res 2007; 9: R15. http://dx.doi.org/10.1186/bcr1648

[15] BENOY I. H., SALGADO R., VAN DAM P., GEBOERS K., VAN MARCK E., et al. Increased serum interleukin-8 in patients with early and metastatic breast cancer correlates with early dissemination and survival. Clin Cancer Res 2004; 10 : 7157-7162. http://dx.doi.org/10.1158/1078-0432.CCR-04$\underline{0812}$

[16] BENDRE M. S., MONTAGUE D. C., PEERY T., AKEL N. S., GADDY D., et al. Interleukin-8 stimulation of osteoclastogenesis and bone resorption is a mechanism for the increased osteolysis of metastatic bone disease. Bone 2003; 33: 28-37. http://dx.doi.org/10.1016/S8756-3282(03)00086-3

[17] ROMAGNANI P., ANNUNZIATO F., LASAGNI L., LAZZERI E., BELTRAME C., et al. Cell cycle-dependent expression of CXC chemokine receptor 3 by endothelial cells mediates angiostatic activity. J Clin Invest 2001; 107: 53-63. http:// dx.doi.org/10.1172/JCI9775

[18] GOLDBERG-BITTMAN L., NEUMARK E., SAGI-ASSIF O., AZENSHTEIN E., MESHEL T., et al. The expression of the chemokine receptor CXCR3 and its ligand, CXCL10, in human breast adenocarcinoma cell lines. Immunol Lett 2004; 92: 171-178. http://dx.doi.org/10.1016/j.imlet.2003.10.020

[19] DATTA D., FLAXENBURG J. A., LAXMANAN S., GEEHAN C., GRIMM M., et al. Ras-induced modulation of CXCL10 and its receptor splice variant CXCR3-B in MDA-MB-435 and MCF-7 cells: relevance for the development of human breast cancer. Cancer Res 2006; 66: 9509-9518. http://dx.doi. org/10.1158/0008-5472.CAN-05-4345

[20] KAWADA K., HOSOGI H., SONOSHITA M., SAKASHITA H., MANABE T., et al. Chemokine receptor CXCR3 promotes colon cancer metastasis to lymph nodes. Oncogene 2007; 26: 4679-4688. http://dx.doi.org/10.1038/sj.onc.1210267

[21] CLARK-LEWIS I., MATTIOLI I., GONG J. H. and LOETSCHER P. Structure-function relationship between the human chemokine receptor CXCR3 and its ligands. J Biol Chem 2003; 278: 289-295. http://dx.doi.org/10.1074/jbc.M209470200

[22] BOOTH V., KEIZER D. W., KAMPHUIS M. B., CLARK-LEWIS I. and SYKES B. D. The CXCR3 binding chemokine IP-10/ CXCL10: structure and receptor interactions. Biochemistry 2002; 41: 10418-10425. http://dx.doi.org/10.1021/bi026020q

[23] MA X., NORSWORTHY K., KUNDU N., RODGERS W. H., GIMOTTY P. A., et al. CXCR3 expression is associated with poor survival in breast cancer and promotes metastasis in a murine model. Mol Cancer Ther 2009; 8: 490-498. http:// dx.doi.org/10.1158/1535-7163.MCT-08-0485

[24] LASAGNI L., FRANCALANCI M., ANNUNZIATO F., LAZZERI E., GIANNINI S., et al. An alternatively spliced 
variant of CXCR3 mediates the inhibition of endothelial cell growth induced by IP-10, Mig, and I-TAC, and acts as functional receptor for platelet factor 4. J Exp Med 2003; 197: 1537-1549. http://dx.doi.org/10.1084/jem.20021897

[25] LUSTER A. D. and LEDER P. IP-10, a -C-X-C- chemokine, elicits a potent thymus-dependent antitumor response in vivo. J Exp Med 1993; 178: 1057-1065. http://dx.doi.org/10.1084/ jem.178.3.1057

[26] DULUC D., CORVAISIER M., BLANCHARD S., CATALA L., DESCAMPS P., et al. Interferon-gamma reverses the immunosuppressive and protumoral properties and prevents the generation of human tumor-associated macrophages. Int J Cancer 2009; 125: 367-373. http://dx.doi.org/10.1002/ ijc. 24401

[27] GROOM J. R. and LUSTER A. D. CXCR3 ligands: redundant, collaborative and antagonistic functions. Immunol Cell Biol 2011; 89: 207-215. http://dx.doi.org/10.1038/icb.2010.158

[28] WALSER T. C., MA X., KUNDU N., DORSEY R., GOLOUBEVA O., et al. Immune-mediated modulation of breast cancer growth and metastasis by the chemokine Mig (CXCL9) in a murine model. J Immunother 2007; 30: 490-498. http:// dx.doi.org/10.1097/CJI.0b013e318031b551

[29] HOLEN I., CROSS S. S., NEVILLE-WEBBE H. L., CROSS N. A., BALASUBRAMANIAN S. P., et al. Osteoprotegerin (OPG) expression by breast cancer cells in vitro and breast tumours in vivo--a role in tumour cell survival? Breast Cancer Res Treat 2005; 92: 207-215. http://dx.doi.org/10.1007/ s10549-005-2419-8

[30] MORONY S., CAPPARELLI C., SAROSI I., LACEY D. L., DUNSTAN C. R., et al. Osteoprotegerin inhibits osteolysis and decreases skeletal tumor burden in syngeneic and nude mouse models of experimental bone metastasis. Cancer Res 2001; 61: 4432-4436.

[31] FILI S., KARALAKI M. and SCHALLER B. Therapeutic implications of osteoprotegerin. Cancer Cell Int 2009; 9(26.

[32] VULCANO M., ALBANESI C., STOPPACCIARO A., BAGNATI R., D'AMICO G., et al. Dendritic cells as a major source of macrophage-derived chemokine/CCL22 in vitro and in vivo. Eur J Immunol 2001; 31: 812-822. http:// dx.doi.org/10.1002/1521-4141(200103)31:3<812::AIDIMMU812>3.0.CO;2-L

[33] GOBERT M., TREILLEUX I., BENDRISS-VERMARE N., BACHELOT T., GODDARD-LEON S., et al. Regulatory T cells recruited through CCL22/CCR4 are selectively activated in lymphoid infiltrates surrounding primary breast tumors and lead to an adverse clinical outcome. Cancer Res 2009; 69: 2000-2009. http://dx.doi.org/10.1158/0008-5472.CAN-08-2360

[34] GUNN M. D., NGO V. N., ANSEL K. M., EKLAND E. H., CYSTER J. G., et al. A B-cell-homing chemokine made in lymphoid follicles activates Burkitt's lymphoma receptor-1. Nature 1998; 391: 799-803. http://dx.doi. org $/ 10.1038 / 35876$

[35] BOWMAN E. P., CAMPBELL J. J., SOLER D., DONG Z., MANLONGAT N., et al. Developmental switches in chemokine response profiles during $\mathrm{B}$ cell differentiation and maturation. J Exp Med 2000; 19: 1303-1318. http://dx.doi. org/10.1084/jem.191.8.1303

[36] PANSE J., FRIEDRICHS K., MARX A., HILDEBRANDT Y., LUETKENS T., et al. Chemokine CXCL13 is overexpressed in the tumour tissue and in the peripheral blood of breast cancer patients. Br J Cancer 2008; 99: 930-938. http://dx.doi. org/10.1038/sj.bjc.6604621

[37] YAU C., ESSERMAN L., MOORE D. H., WALDMAN F., SNINSKY J., et al. A multigene predictor of metastatic outcome in early stage hormone receptor-negative and triplenegative breast cancer. Breast Cancer Res 2010; 12: R85. http:// dx.doi.org/10.1186/bcr2753

[38] MARTINEZ F. O., HELMING L. and GORDON S. Alternative activation of macrophages: an immunologic functional perspective. Annu Rev Immunol 2009; 27: 451-483. http:// dx.doi.org/10.1146/annurev.immunol.021908.132532

[39] ZOHNY S. F. and FAYED S. T. Clinical utility of circulating matrix metalloproteinase-7 (MMP-7), CC chemokine ligand 18 (CCL18) and CC chemokine ligand 11 (CCL11) as markers for diagnosis of epithelial ovarian cancer. Med Oncol 2010; 27: 1246-1253. http://dx.doi.org/10.1007/s12032-009-9366-X

[40] LEUNG S. Y., YUEN S. T., CHU K. M., MATHY J. A., LI R., et al. Expression profiling identifies chemokine (C-C motif) ligand 18 as an independent prognostic indicator in gastric cancer. Gastroenterology 2004; 127: 457-469. http://dx.doi. org/10.1053/j.gastro.2004.05.031

[41] CHEN J., YAO Y., GONG C., YU F., SU S., et al. CCL18 from tumor-associated macrophages promotes breast cancer metastasis via PITPNM3. Cancer Cell 2011; 19: 541-555. http:// dx.doi.org/10.1016/j.ccr.2011.02.006

[42] MCQUIBBAN G. A., GONG J. H., TAM E. M., MCCULLOCH C. A., CLARK-LEWIS I., et al. Inflammation dampened by gelatinase A cleavage of monocyte chemoattractant protein-3. Science 2000; 289: 1202-1206. http://dx.doi.org/10.1126/ science.289.5482.1202

[43] DESHMANE S. L., KREMLEV S., AMINI S. and SAWAYA B. E. Monocyte chemoattractant protein-1 (MCP-1): an overview. J Interferon Cytokine Res 2009; 29: 313-326. http:// dx.doi.org/10.1089/jir.2008.0027

[44] LEBRECHT A., GRIMM C., LANTZSCH T., LUDWIG E., HEFLER L., et al. Monocyte chemoattractant protein-1 serum levels in patients with breast cancer. Tumour Biol 2004; 25(1-: 14-17.

[45] CONTII. and ROLLINSB.J. CCL2 (monocytechemoattractant protein-1) and cancer. Semin Cancer Biol 2004; 14: 149-154. http://dx.doi.org/10.1016/j.semcancer.2003.10.009 


\section{Supplementary Information}

\section{Altered levels of plasma chemokines in breast cancer and their association with clinical and pathological characteristics}

D. NARITA ${ }^{1,2}$, E. SECLAMAN ${ }^{1}$, A. ANGHEL ${ }^{1}$, R. ILINA ${ }^{3}$, N. CIREAP ${ }^{3}$, S. NEGRU ${ }^{4}$, I. O. SIRBU ${ }^{1}$, S. URSONIU ${ }^{5, *}$, C. MARIAN $^{1, *}$

${ }^{1}$ Department of Biochemistry, University of Medicine and Pharmacy "Victor Babes", Timisoara, Romania; ${ }^{2}$ Institute for Laboratory Diagnostic and Transfusion Medicine, Donauisar Klinikum, Deggendorf, Germany; ${ }^{3}$ Department of Surgical Oncology, University of Medicine and Pharmacy "Victor Babes", and Municipal Hospital, Timisoara, Romania; ${ }^{4}$ Department of Oncology, University of Medicine and Pharmacy "Victor Babes", and Oncomed Oncology Practice, Timisoara, Romania; ${ }^{5}$ Department of Public Health, University of Medicine and Pharmacy "Victor Babes", Timisoara, Romania

*Correspondence: sursoniu@umft.ro,cmarian@umft.ro 


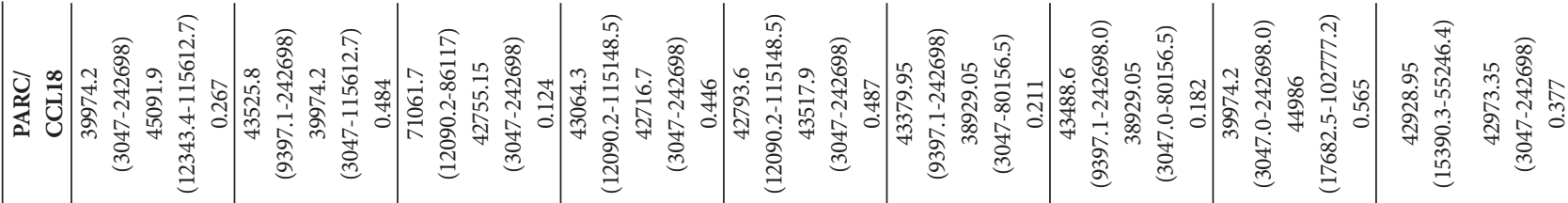

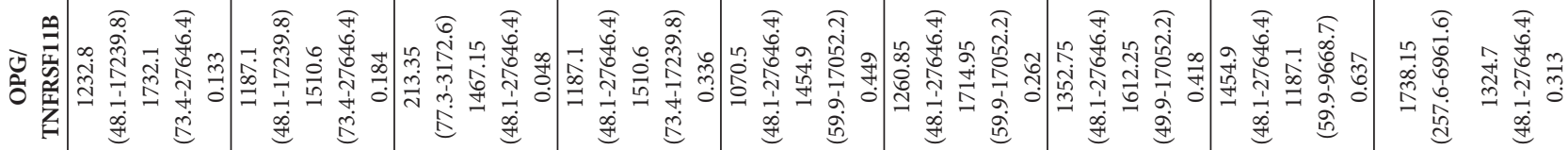

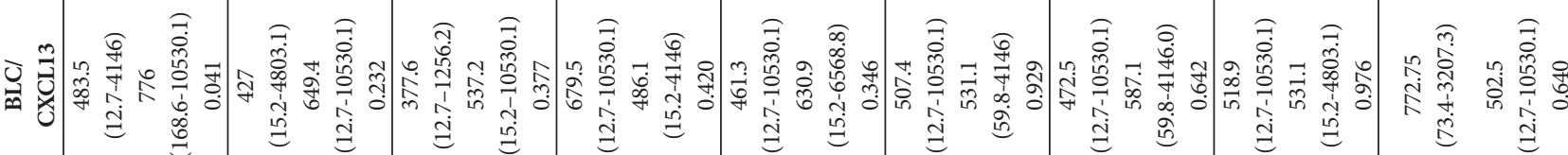

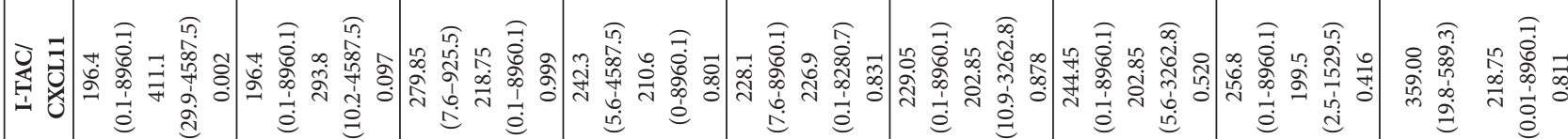

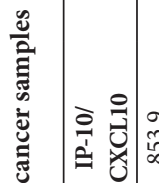

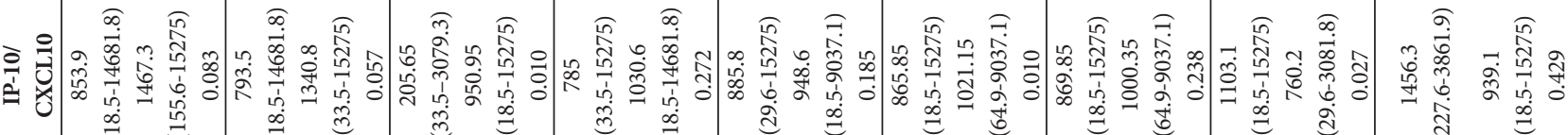

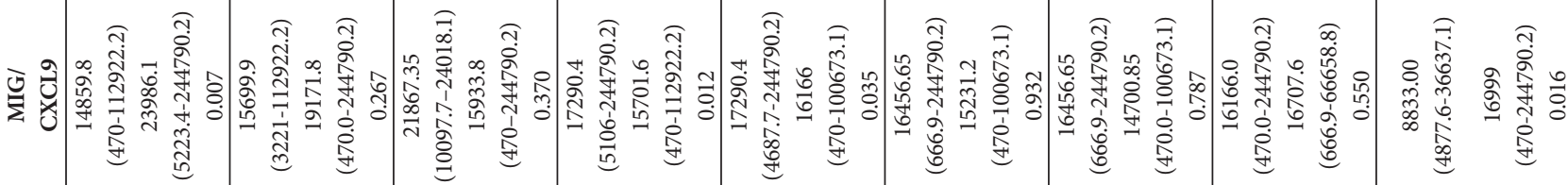

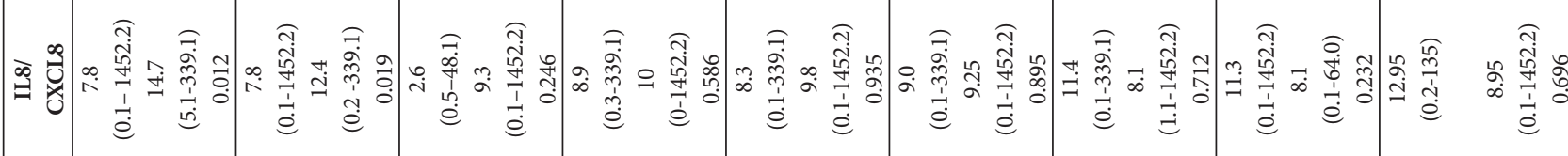

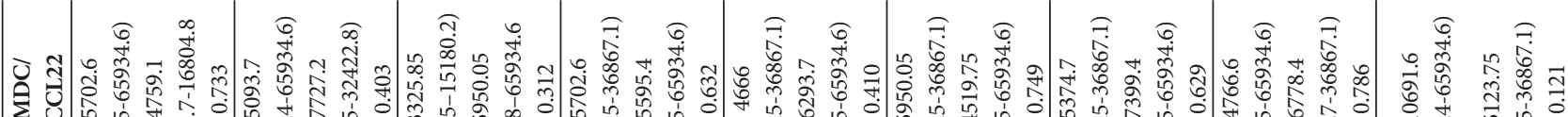

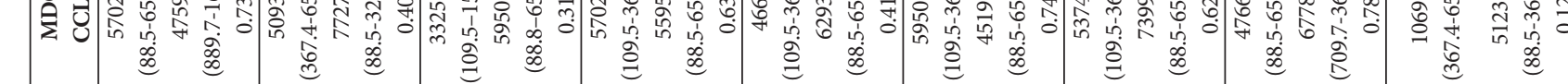

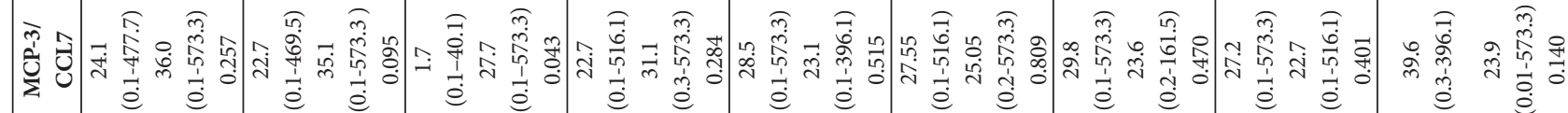

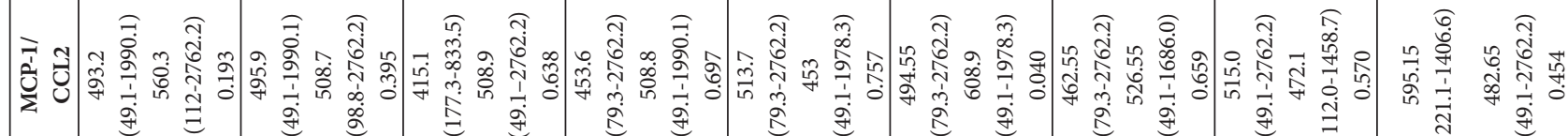

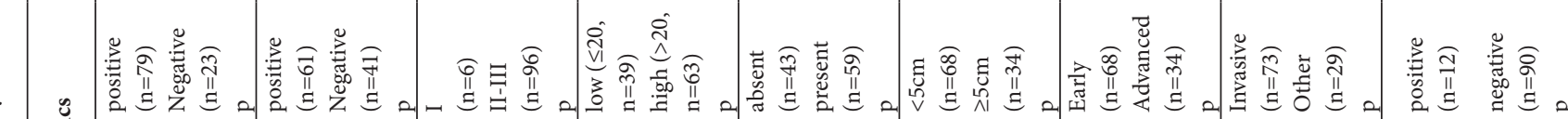

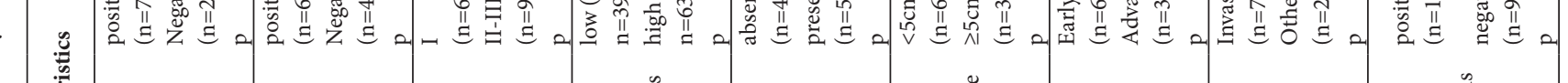

